Check for updates

\title{
The Impact of use of Double Set-up on Infection Rates in Revision Total Knee Replacement and Limb Salvage Procedures
}

Jennifer A. Waterman, DO*; Jon E. Minter, DO**; Paul J. Ghattas, DO*; Brandon M. Green, DO*

\begin{abstract}
A retrospective analysis was performed to determine the impact of utilizing a double set-up procedure on reducing infection rates revision total knee and limb salvage procedures in patients with known joint infection. Eighteen cases fit selection criteria. The recurrence rate of infection was 5.5\% which is less than reported recent literature review. This suggests the use of a double set-up in combination with other infection reducing protocols may help further reduce recurrent infection.
\end{abstract}

Keywords: double set-up, infection, revision total knee arthroplasty, limb-salvage

\section{Introduction}

Infection of an existing total knee arthroplasty (TKA) continues to be one of the most devastating complications associated with these procedures. Infection is costly [1] and has significant associated co-morbidities. Bannister et al estimated "that an infected hip replacement in modern practice cost the equivalent of five primary procedures [2]. Primary TKA infection rates are reported in recent literature ranging from $0.3 \%-3 \%$ cases [3]. A historic incidence of TKA infection is as high as $23 \%$ [4]. Unfortunately, deep infection rates increase in those undergoing revision surgeries to address previous infected TKA [1], and failure due to infection has been reported as high as $46 \%$ in some studies [5]. Infection is the most common cause of the revision implant failure [6]. There has been a focus on meth-

* Wellmont Orthopedic Residency Program, 130 W. Ravine Rd., Kingsport, TN 37660

** Northside Total Joint Specialists, 3400-C Old Milton Pkwy., Suite 290, Alpharetta, GA 30005

(C) 2015 Jennifer A. Waterman, Jon E. Minter, Paul J. Ghattas, Brandon M. Green. All rights reserved DOI: 10.15438/rr.5.1.98 • ISSN 2331-2262 (print) • ISSN 2331-2270 (online)

For complete copyright and licensing information please refer to the end of this article. ods to reduce infection rates to include: mitigation of risk factors, use of operating room laminar flow, surgical team space suits, pre-operative workup prior to revision, staged procedures, use of antibiotic spacers and cement, antibiotic regimens, sonication [7], intra-operatives culture protocols among many other methods. As Cierny and Mader defined a staging system of the host based on medical conditions, systemic and local, that also impacts outcomes of surgical intervention to eradicate infection. In this staging system A-hosts are healthy, B-hosts are compromised by one or more local or systemic parameters, and C-hosts are not considered aggressive surgical candidates. In 2002 Cierny and DiPasquale also described the use of double set up for a total of 43 patients treated for total joint prosthetic infections with survival rate of $100 \%$ in type A hosts, $86 \%$ in B-hosts, and $0 \%$ of type C-hosts $[8,9,10]$. The use of a double set-up procedure in addition to existing accepted operative procedures and its impact on reducing revision infection reoccurrence has not been widely been investigated in the literature.

This series describes a novel technique that may be helpful in recalcitrant infection. The double set-up proce- 
dure was utilized for patients presenting for initial treatment and subsequent reimplantation. The Cierny system was utilized to label hosts to provide treatment specific to their disease process. This paper also provides details on the sequence of surgical steps required to successfully perform this technique.

The purpose of this study is to describe a novel technique that may help decrease recurrent infection in previously infected revision joint surgery and determine the impact of utilizing a double set-up procedure on reducing infection rate in revision total knee and limb salvage procedures performed for existing infected joints. I hypothesize that double set-up in combination with already accepted protocols to include preoperative infection screening (erythrocyte sedimentation rate, c-reactive protein, complete blood count, and joint aspiration) and intraoperative cultures (frozen section, aerobic, anaerobic, fungal, and acid fast bacilli cultures) will reduce incidence of infections in the revision setting. It is our hypothesis that the double set-up with aggressive surgical debridement an important factor in reduction of recurrent infection. In this case series, we show that the double set-up reduces infections rates in patients undergoing revision surgery for infection.

\section{Methods}

A retrospective analysis of medical records was performed. Inclusion criteria was any revision total knee arthroplasty or limb salvage procedures performed at our facility utilizing the double set-up procedure between the dates of July 1, 2008 through May 1, 2012. Pre-operative diagnoses of all 18 patients included infected total joint prosthesis. Cases using double set-up in joints other than the knee were excluded from the retrospective analysis.

A double set-up procedure involves the use of two separate sterile instrument sets, the first used for the initial debridement and resection and the second used for re-implantation or second portion of the procedure if re-implantation is not performed. This technique is utilized to address revision of previously infected joints. At no time are instruments from the initial debridement used in the second portion of the procedure. The procedure begins with extensive and thorough debridement; the incision is first carried down through the subcutaneous tissue to the level of the articular/capsular level. Devitalized and fibrous hypertrophic tissue is entirely debrided. The debridement is continued deeper and includes the previous antibiotic spacer (if present), cement or hardware remaining. Suspicious bone and surrounding tissues and bone canals are debrided. Bone saucerization and resection are also performed based on findings. Tissue specimens are collected and sent to pathology for evaluation for signs of acute inflammation (ie. Frozen section) Della Valle et al in their retrospective review of 64 two-stage arthroplasties reported that intraoperative analysis of frozen sections at time of reimplantation had sensitivity of $25 \%$, a specificity of $98 \%$, a negative predictive values of $95 \%$, and accuracy rate of $94 . \%$ They used a mean of $>10$ polymorphonuclear leukocytes (PMN) per high-power field in the five most cellular sites examined as a positive test for acute inflammation [11]. Bori et al utilized the Feldman Criterion (more than five PMN per highpower field in the five most cellular fields and the presence of at least one PMN per high-power field identified in 10 cellular fields) and found that frozen sections have a sensitivity of $28.5 \%$, specificity $100 \%$, positive predictive value $100 \%$, and negative predictive value of $73.6 \%$ [12]. The high specificity and positive predictive value of this makes this criterion a strong predictor the presence of persistent infection. Periprosthetic tissue specimen of the soft tissue, bone-cement interface or the pseudocapsule, were considered positive for active infection if there were more than five polymorphonuclear leukocytes per high-power field. The wound is then copiously irrigated with pulsatile lavage using a Clorpactin trademark by Unite-Guardian Inc Hauppauge solution. Following the entire sequence of debridement and irrigation the wound is then packed with Clorpactin soaked sponges prior to wound capsule and skin closure. If no signs of acute inflammation are detected reimplantation can be performed. All members of the operative team perform complete change of gloves, gowns and sterile preparation. The patient is draped again in the standard sterile fashion and all new sterile instrumentation is used for the second portion of the case. Approximate turnaround time was 17 minutes between cases.

Statistical analysis was performed and 95\% confidence intervals were calculated using the Clopper-Pearson intervals formula assuming binomial distribution. CP-confidences intervals were calculated for other studies that reported on 2-stage revision techniques aimed to prevent infection. (See Table 1)

\section{Results}

The patients were first identified by reviewing records and pulling charts on all patients with current procedural terminology (CPT) code 27599 which is associated with double set-up procedure in time period of October 2008 through April 2012. A total of 79 patients were identified and then this was narrowed to include on those pro- 
Table 1. Patient Data

\begin{tabular}{|c|c|c|c|c|c|c|}
\hline Patient & $\begin{array}{l}\text { Age at } \\
\text { last } S x\end{array}$ & Sex & Host Classification & $\begin{array}{c}\text { Number of Revision } \\
\text { Surgeries }\end{array}$ & Final Surgery & Time from final surgery as $7 / 1 / 13$ \\
\hline 1 & 55 & $\mathrm{~F}$ & B systemic & 2 & Hinged total knee Depuy limb salvage system & 27months 2 weeks \\
\hline 3 & 62 & $\mathrm{~F}$ & A & 2 & Limb salvage system & 15months 1 week \\
\hline 4 & 64 & $\mathrm{M}$ & $\mathrm{B}$ & 2 & Depuy TC3 & 14months 2 weeks \\
\hline 7 & 73 & $\mathrm{M}$ & B systemic & 1 & Arthrodesis & 30months 2 weeks \\
\hline 8 & 72 & $\mathrm{~F}$ & B systemic & 3 & Hinged total knee Depuy limb salvage system & 15months 1week \\
\hline 9 & 59 & $\mathrm{~F}$ & A & 2 & Depuy TC3 & 40months 2 weeks \\
\hline 10 & 67 & $\mathrm{~F}$ & A & 3 & Depuy Limb salvage system & 23months \\
\hline 14 & 66 & $\mathrm{~F}$ & B systemic & 2 & Depuy TC3 & 15 months \\
\hline 15 & 78 & M & B systemic & 2 & Depuy TC3 & 51months 2 weeks \\
\hline 16 & 66 & $\mathrm{~F}$ & A & 2 & Depuy TC3 & 25 months 3 weeks \\
\hline 17 & 54 & M & B systemic & 1 & Polyethylene exchange & 49months 3 weeks \\
\hline 18 & 41 & $\mathrm{M}$ & B systemic & 1 & Polyethylene exchange & 33 months 3 weeks \\
\hline
\end{tabular}

Table 2. Antibiotic Regimen

\begin{tabular}{|c|c|c|c|}
\hline PT & $\begin{array}{c}\text { Antibiotic treatment prior } \\
\text { to final surgery }\end{array}$ & $\begin{array}{l}\text { Peri-op antibiotic } \\
\text { final surgery }\end{array}$ & $\begin{array}{l}\text { Infectious Disease } \\
\text { Consult }\end{array}$ \\
\hline 1 & $\begin{array}{l}\text { doxycycline/trimethoprim- } \\
\text { sulfamethoxazole }\end{array}$ & cefazolin/cefipime & Yes \\
\hline 2 & ceftriaxone/flagyl & vancomycin & Yes \\
\hline 3 & daptomycin & vancomycin & Yes \\
\hline 4 & none & cefazolin & No \\
\hline 5 & minocycline & vancomycin & Yes \\
\hline 6 & vancomycin & vancomycin & Yes \\
\hline 7 & vancomycin & daptomycin & Yes \\
\hline 8 & daptomycin & vancomycin & Yes \\
\hline 9 & piperacillin/tazobactam & cefazolin & Yes \\
\hline 10 & daptomycin & vancomycin & Yes \\
\hline 11 & vancomycin & cefepime & Yes \\
\hline 12 & vancomycin & vancomycin & Yes \\
\hline 13 & None & cefazolin & No \\
\hline 14 & rifampin & vancomycin & Yes \\
\hline 15 & None & cefazolin & No \\
\hline 16 & $\begin{array}{l}\text { vancomycin piperacillin/ } \\
\text { tazobactam }\end{array}$ & vancomycin & Yes \\
\hline 17 & $\begin{array}{l}\text { vancomycin/rifampin/ } \\
\text { piperacillin/tazobactam }\end{array}$ & $\begin{array}{l}\text { piperacillin/ } \\
\text { tazobactam }\end{array}$ & Yes \\
\hline 18 & $\begin{array}{l}\text { piperacillin/tazobactam } \\
\text { vancomycin }\end{array}$ & $\begin{array}{l}\text { piperacillin/ } \\
\text { tazobactam / } \\
\text { vancomycin }\end{array}$ & Yes \\
\hline
\end{tabular}

cedures involving the knee and excluding hip procedures. The charts of the remaining knee patients then were carefully reviewed to confirm a double set-up procedure was performed to address infection. In this retrospective analysis a total of 18 cases were identified that fit selection criteria. All patients belonged to a single fellowship trained total joint surgeon. The breakdown of women to men was 11 to 7 respectively. Ages at final surgery ranged from 41 to 80 years with a mean of 61 years. Using the Cierny/Mader host classification five were A-hosts, 12 patients were B-host, one patient was a C-host. Perioperative antibiotics were utilized in every case (Table 2 ). Fifteen patients completed an antibiotic course prior to their definitive surgical intervention as prescribed by infectious disease specialist. Four cases were single revision procedures using a double set-up. Ten patients underwent two-stage revisions utilizing the double set-up. Three patients had a total of three revision surgeries to address their initial infection prior to definitive reimplantation or final surgery. One patient required a total of four surgeries, the third surgery a result of failed hardware in arthrodesis and the most recent surgery was an amputation with recurrent infection. The patient who required amputation was the only C-host in our study. This is consistent with Cierny's work in which no C-host's had successful resolution of infection in C-host patients. At the time of this publication only one patient of $18(5.5 \%)$ had developed recurrent infection and no patients had expired. Eight patients had revision components 
using the Depuy TC3, Table 3.

three patients underwent polyethylene swap only, one patient kept an arthrodesis as final treatment, and five had Depuy limb salvage systems implanted. Time out from final surgery ranged from 13 months to 51 months 2 weeks with mean time out 28

\begin{tabular}{|c|c|c|}
\hline & No Infection & Total \\
\hline Waterman & 17 & \\
\hline \multirow[t]{2}{*}{ Gooding } & 101 & \\
\hline & 12 & \\
\hline Kubista & 310 & \\
\hline Macheras & 31 & \\
\hline McPherson & 59 & \\
\hline Mortazavi & 68 & \\
\hline Mortazavi (B & 69 & \\
\hline \multirow[t]{2}{*}{ Sorli } & 37 & \\
\hline & 4 & 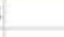 \\
\hline \multicolumn{3}{|l|}{ Suarez } \\
\hline Cierny & 30 & \\
\hline
\end{tabular}

\begin{tabular}{|r|r|}
\hline 18 & $95 \%$ \\
\hline 115 & $95 \%$ \\
\hline 14 & $95 \%$ \\
\hline 368 & $95 \%$ \\
\hline 34 & $95 \%$ \\
\hline 70 & $95 \%$ \\
\hline 91 & $95 \%$ \\
95 & $95 \%$ \\
55 & $95 \%$ \\
\hline 11 & $95 \%$ \\
\hline
\end{tabular}

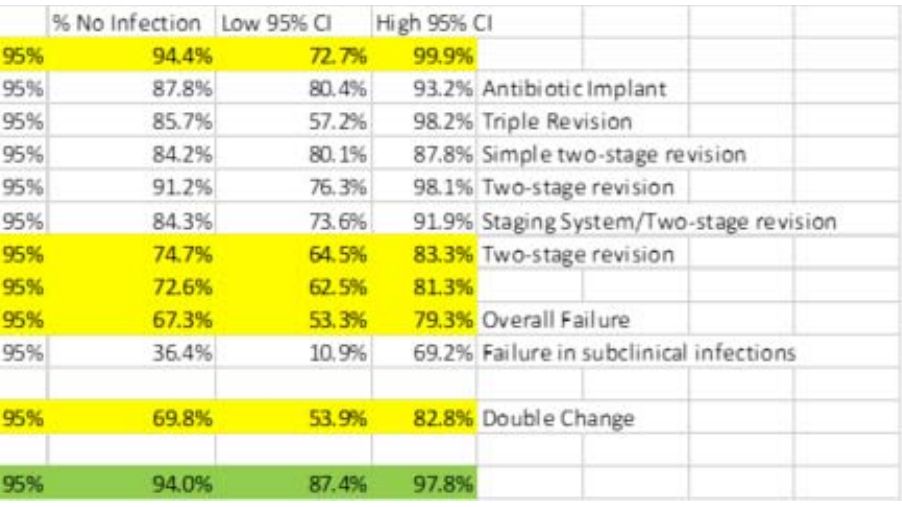
months and a median 27 months. In the cases of two stage treatment time range from initial temporary arthrodesis to reimplantation was a mean of six months.

\section{Discussion}

The recurrence rate of infection in this study was 5.5\% which is less than that expected in the management of the infected TKA, and as discussed previously recurrence rate after revision has been quoted much higher than this. Therefore, patients in our case series have lower infection rates than those quoted in recent publications. Within our literature review our case series most closely mirrors Cierny's studies. The Cierny study used a double set up procedure but had higher infection rates than found in our retrospective following revision surgery. In Cierny's work infection reoccurrence occurred in all $\mathrm{C}$ patients and in some $\mathrm{B}$ patients [8]. Our results showed no infection reoccurrence in B patients and our only failure was in our single C-host. This would suggest that the double set-up procedure with aggressive surgical debridement used in combination with other infection reducing protocols such as intraoperative cultures and postoperative antibiotic regimens may help further reduce recurrent infection in recalcitrant infectious revision cases. Sorili's work showed that explanted antibacterial spacers were colonized at the second stage, which implies the sterile field is no longer sterile after removal; this provides further evidence and motivation to utilize a double set-up. [13]. Table 3 demonstrates confidence intervals between our study and multiple studies examining reinfection rates in revision surgery. In Mortazavi's work infection in revision surgery was tenfold higher than in primary TKA, after retrospective review of 499 TKA revisions 102 (18\%) required re-revision with infection being the most common cause (445). [14] Further comparing our study to Sorli and Mortazavi with Fisher's exact test we can show a Fisher's exact test of 0.020 which is statisti-

cally significant and 0.0676 respectively which is nearly statistically significant (Table 4) this also supports that the use of a double set up may significantly impact infection in revision TKA surgery.

Limitations of this study are related to being a retrospective analysis which by default can cause data bias. This is a small case series, with only 18 cases. An increased power 100 patients with a $4 \%$ infection rate would indicate clinically significant improvement in infection rates. There are

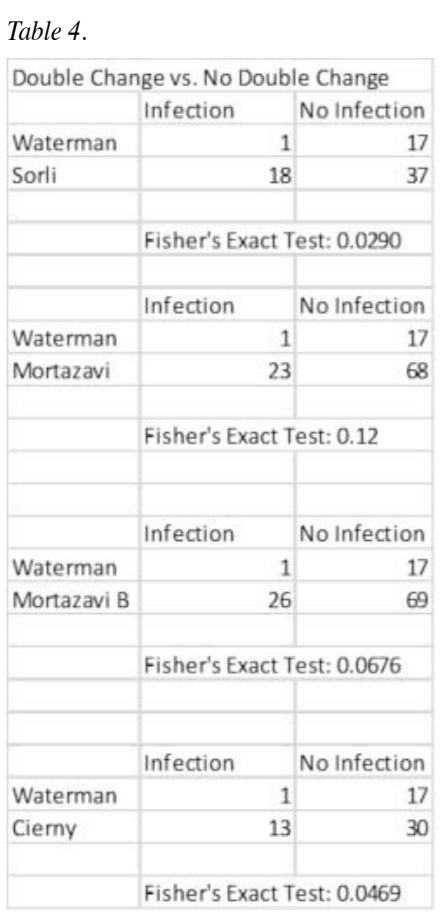
confounding variables as the patients in the series underwent different surgeries; polyexchange, Depuy TC3, limb salvage, arthrodesis, and amputation. Another limitation is not having a control group without a double-set up for comparison. A potential weakness is that our study did not include total hip arthroplasty, however, limiting this study to total knees did allow us control variables between these types of surgeries.

A future direction would be to consider a prospective review of infection rate/recurrence in revision TKA performed using a double set up procedure and compare that to our present results to determine significance and further plausibility of this technique which the senior author has utilized for years.

\section{Author's Note:}

This paper is dedicated to the memory and legacy of George Cierny, M.D. 


\section{References}

1. Mortazavi, S; Schwartzenberger, J; Austin, M; Purtill, J; Parvizi, J. Revision total knee arthroplasty infection. Clinical Orthopedic Related Research 2010 468:2052 2059.

2. Bannister, G. Prevention of infection in joint replacement. Current Orthopaedics. 2002; 16 (6): 426-433.

3.

4. Citak, M; Gessman, J; Fehemer, T; Russe, O; Schildhauer, T, Seybold, D. Twostage revision of infected total knee arthroplasty using a distraction spacer. Technology and Health Care 19 2011; 167-171

5. Macheras, G; Kateros, K; Galanakos, S; Koutsostathis, S; Kontou, E. The longterm results of two-stage protocol for revision of an infected total knee replacement. Journal of Bone and Joint Surgery British Volume. 2011 Nov; 93 (11): 1487 92.

6. Suarex, J; Griffin, W; Springer B; Fehring T; Mason JB; Odum S. Why do revision knee arthroplasties fail? J Arthroplasty. 2008: 23: 99-103

7. Parvizi, J; Zmistowski, B, Adeli, B. Periprosthetic joint infection treatment options. Orthopedics 2010 Sept Vol 33 issue 9.

8. Sorli, L; Puig, L; Torres-Claramunt, R; Gonzales, A; Alier, A; Knobe. The relationship between microbiology results in the second of a two-stage exchange procedure using cement spacers and the outcome after total joint replacement for infection. The Journal of Bone and Joint Surgery British volume. 2012 Feb;94 (2): 249-53.

9. Cierny, G. Periprosthetic Total Joint Infections. Staging, Treatment, and Outcomes. Clinical Orthopaedics and Related Research. 403. pp23-28.

10. Cierny, G. Procedure-Related Reduction of the Risk of Infection. OKU: Chapter 4 Musculoskeletal Infection
11. Cierny, G. Mader, J. The Classic. A Clinical Staging System for Adult Osteomyelitis. Clinical Orthopaedics and Related Research.

12. Della, V; Parvizi, J; Bauer, TW. American Academy of Orthopaedic Surgeons American Academy of Orthopaedic Surgeons clinical practice guideline on The diagnosis of periprosthetic joint infections of the hip and knee. J Bone Joint Surg Am 2011;93(14): 1355-1357

13. Bori, G; Soriano, A; Garcia, S; Mallofre, C; Riba, J; Mesna, J Usefulness of histological analysis for predicting the prescence of microorgansims at the time of reimplantation after hip resection arthroplasty for treatment of infection. J Bone Joint Surg Am 1999;81(5):684-689

14. Sorli, L; Puig, L; Torres-Claramunt, R; Gonzales, A; Alier, A; Knobe. The relationship between microbiology results in the second of a two-stage exchange procedure using cement spacers and the outcome after total joint replacement for infection. The Journal of Bone and Joint Surgery British volume. 2012 Feb;94 (2): 249-53.

15. Mortazavi, S; Schwartzenberger, J; et al. Revision Total Knee Arthroplasty Incidence and Predictors. Clin Orthop Relat Res (2010) 468:2052-2059

\section{Copyright \& Licensing}

Authors retain copyright and grant the journal right of first publication with the work. Reconstructive Review follows the Creative Commons Attribution-NonCommercial CC BY-NC. This license allows anyone to download works, build upon the material, and share them with others for non-commercial purposes as long as they credit the senior author, Reconstructive Review, and the Joint Implant Surgery \& Research Foundation (JISRF). An example credit would be: "Courtesy of (senior author's name), Reconstructive Review, JISRF, Chagrin Falls, Ohio". While works can be downloaded and shared they cannot be used commercially.

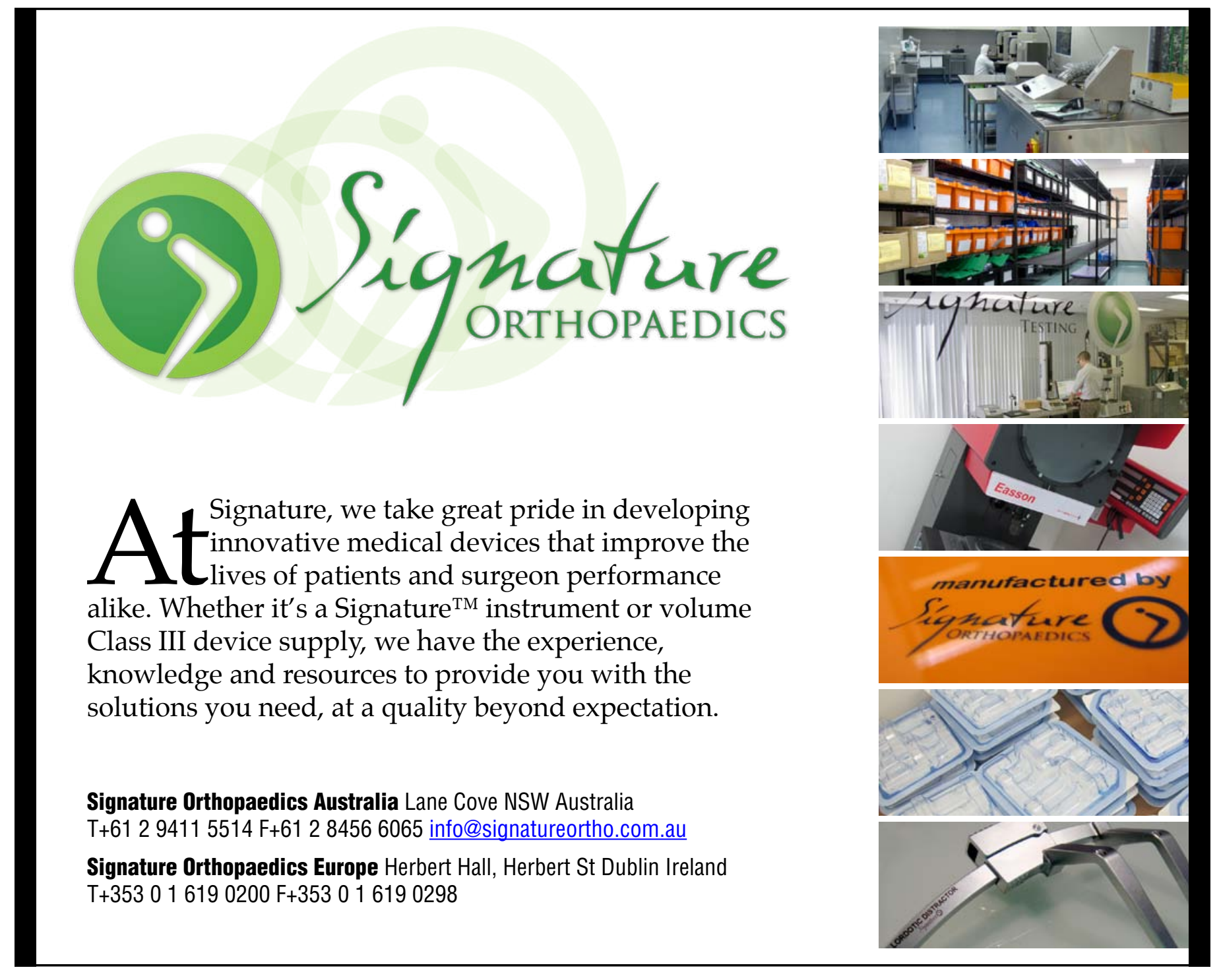

